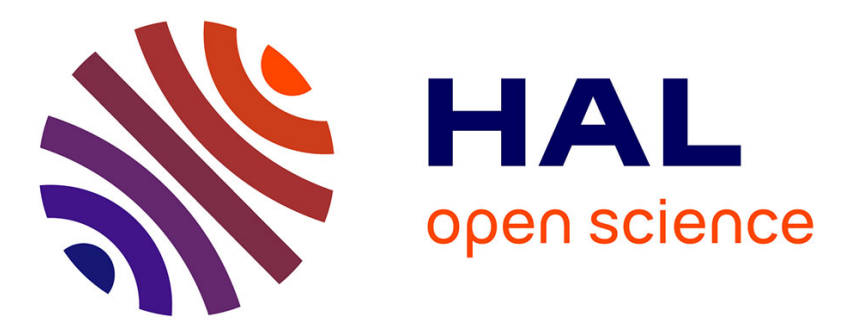

\title{
Modelisation des composants a betérostructures AlGaAs/GaAs MIS-Like-FET et DMt par la methode Monte Carlo
}

K. Bellahsni, Jean-Luc Thobel, P. Bourel, R. Fauquembergue, M. Pernisek

\section{- To cite this version:}

K. Bellahsni, Jean-Luc Thobel, P. Bourel, R. Fauquembergue, M. Pernisek. Modelisation des composants a betérostructures AlGaAs/GaAs MIS-Like-FET et DMt par la methode Monte Carlo. Journal de Physique III, 1991, 1 (4), pp.521-529. 10.1051/jp3:1991136 . jpa-00248596

\section{HAL Id: jpa-00248596 https://hal.science/jpa-00248596}

Submitted on 1 Jan 1991

HAL is a multi-disciplinary open access archive for the deposit and dissemination of scientific research documents, whether they are published or not. The documents may come from teaching and research institutions in France or abroad, or from public or private research centers.
L'archive ouverte pluridisciplinaire $\mathbf{H A L}$, est destinée au dépôt et à la diffusion de documents scientifiques de niveau recherche, publiés ou non, émanant des établissements d'enseignement et de recherche français ou étrangers, des laboratoires publics ou privés. 
Classification

Physics Abstracts

$85.30 \mathrm{~T}-85.30 \mathrm{D}-72.80 \mathrm{E}$

\title{
Modélisation des composants à hétérostructures AlGaAs/GaAs MIS-Like-FET et DMT par la méthode Monte Carlo
}

\author{
K. Bellahsni, J. L. Thobel, P. Bourel, R. Fauquembergue et M. Pernisek \\ Centre Hyperfréquences et Semiconducteurs UA CNRS 287, Université des Sciences et \\ Techniques de Lille Flandres Artois, 59655 Villeneuve D’Ascq Cedex, France
}

(Reçu le 30 avril 1990, révisé le 23 juillet 1990, accepté le 27 août 1990)

\begin{abstract}
Résumé. - Nous présentons dans ce travail des résultats obtenus par une modélisation Monte Carlo bidimenisionnelle du MIS-Like-FET AlGaAs/GaAs intrinsèque qui tient compte du courant grille. Nous décrivons les phénomènes physiques (champ, énergie moyenne) intervenant dans le fonctionnement du MIS-Like-FET autoaligné à faibles et fortes tensions de grille positives. Une conductance différentielle négative apparaît à fortes tensions de grille et son amplitude est sensible à divers paramètres technologiques. En particulier, elle diminue lorsqu'on augmente la distance source-grille $L_{\mathrm{sg}}$ et la résistánce de métallisation de grille $R_{\mathrm{g}}$. Le MIS-Like-FET polarisé à des tensions de grille modérées présente un courant drain trop faible pour son application en puissance, nous proposons aussi des résultats obtenus en simulant le MIS-Like-FET à canal dopé (DMT) et nous remarquons que le courant drain est fortement augmenté.
\end{abstract}

\begin{abstract}
AlGaAs/GaAs MIS-Like-FET has been simulated using a two dimensional ensemble Monte Carlo model, accounting for gate current. The physical phenomena involved in the selfaligned device's behaviour are discussed. Electrical characteristics have been calculated and for high $V_{g s}$, a negative differential conductance has been found, the magnitude of which is very dependent on source-gate spacing and gate resistance. The low value of drain current obtained for möderate gate bias is a major drawback for power application. This limitation can be overcome using a doped GaAs channel that leads to a new device called "DMT ". A DMT structure has also been simulated and drain current has been found much higher than in conventional MISLike-FET.
\end{abstract}

\section{Introduction.}

Le MIS-Like-FET AlGaAs/GaAs intrinsèque a fait l'objet ces dernières années d'un intérêt particulier parce qu'il se révèle être un composant présentant des potentialités intéressantes dans de nombreux domaines (logique, oscillateurs, etc...).

Les avantages offerts par ce composant sont, d'une part, l'absence d'effet lié aux centres DX dans les couches non dopées et, d'autre part la bonne uniformité de la tension seuil qui font de lui un candidat privilégié pour la conception de circuits intégrés.

Néanmoins, la faible hauteur de la barrière de potentiel $\Delta E_{\mathrm{c}}$ à l'interface $\mathrm{Al}_{x} \mathrm{Ga}_{1-x} \mathrm{As} / \mathrm{GaAs}$ (environ $0,35 . \mathrm{eV}$, pour $x=0,45$ ) peut faire apparaître un courant grille qui est néfaste à certaines applications mais souhaitable pour l'obtention d'une résistance 
différentielle négative. Nous proposons dans ce travail l'étude complète de MIS-Like-FET typiques afin de dégager les aspects fondamentaux des différents modes de fonctionnement et l'influence de certains paramètres tels que la distance source-grille, la résistance de grille et le dopage du canal.

\section{Modèle.}

Pour simuler le MIS-Like-FET, nous avons utilisé la méthode de Monte Carlo [1] associée à une résolution bidimensionnelle de l'équation de Poisson, par la technique de Hockney [2].

Afin de décrire la dynamique électronique dans les matériaux AlGaAs et GaAs, nous considérons que leur bande de conduction est du type 3 vallées $(\Gamma, \mathrm{L}, \mathrm{X})$ isotropes, non paraboliques. Nous tenons compte des interactions sur phonons optique polaire et optique non polaire, acoustique, piézoélectrique, intervallées, désordre d'alliage (pour l'AlGaAs). L'interaction sur impuretés ionisées est traitée dans l'approximation de Brooks Herring [3]. Les paramètres matériau sont puisés dans la littérature [4]. La quantification de l'énergie dans la zone d'accumulation n'est pas prise en compte, les vitesses des porteurs n'en sont pas affectées, au moins à température ambiante [5, 6]. L'interface AlGaAs/GaAs est considérée comme une barrière de potentiel et le passage des porteurs d'un matériau à l'autre se fait en respectant la conservation de l'énergie totale et de la composante du vecteur d'onde parallèle à l'interface. Nous négligeons le transfert d'électrons par effet tunnel car l'épaisseur de la couche $\mathrm{AlGaAs}$ reste supérieure à $200 \AA$ [7]. La condition d'ohmicité $n=N_{\mathrm{d}}$ est imposée au niveau des contacts de source et de drain. En ce qui concerne le contact de grille le décompte des porteurs, qui y sont collectés, permet le calcul du courant grille.

Dans ce qui suit nous considérons la tension de grille interne $V_{\mathrm{gi}}$ effectivement appliquée au semiconducteur :

$$
V_{\mathrm{g} 1}=V_{\mathrm{gs}}-\Phi_{\mathrm{b}}
$$

où la hauteur de barrière de Schottky $\Phi_{\mathrm{b}}$ est posée égale à $1,1 \mathrm{eV}[8]$.

\section{Etude d'une structure auto-alignée.}

La structure simulée est composée, comme on peut le voir figure 1, d'un substrat GaAs non dopé d'épaisseur $0,6 \mu \mathrm{m}$ sur lequel est déposée une couche d'AlGaAs non dopée de $400 \AA$, surmontée d'une grille métallique de longueur $1 \mu \mathrm{m}$; les contacts de source et de drain sont dopés à $10^{18} \mathrm{~cm}^{-3}$

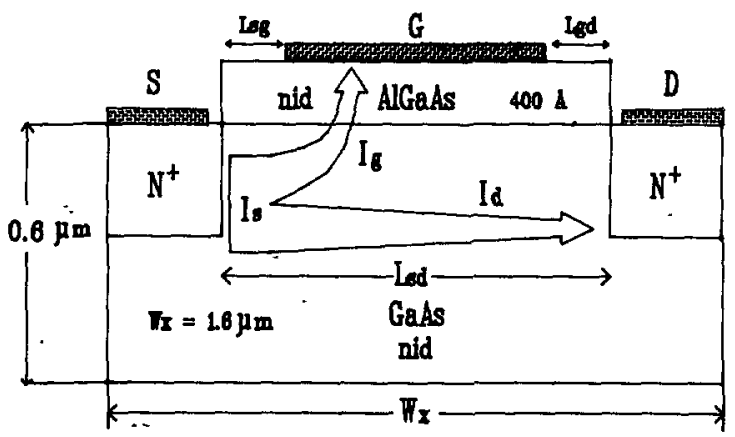

Fig. 1. - Structure bidimensionnelle simulée de MIS-Like-FET AlGaAs/GaAs.

[Simulated structure of AlGaAs/GaAs MIS-Like-FET.] 
Le MIS-Like-FET AlGaAs/GaAs fonctionne en régime d'enrìchissement, à polarisation de grille positive, les électrons provenant des contacts ohmiques de source et de drain s'accumulent dans le puits de potentiel à l'interface AlGaAs/GaAs.

Sur les figures 2 et 3 qui représentent l'énergie moyenne des porteurs et le champ perpendiculaire à l'interface pour une tension grille faiblement positive $\left(V_{\mathrm{gi}}=0,4 \mathrm{~V}\right)$ et pour différentes valeurs de la tension drain $\left(V_{\mathrm{ds}}=0,5,1,0,2,0 \mathrm{~V}\right)$. Nous observons sur ces figures que l'énergie n'est supérieure à $\Delta E_{\mathrm{c}}(\# 0,35 \mathrm{eV})$ qu'en région de fin de grille là où le champ transverse devient négatif, c'est-à-dire lorsqu'il s'oppose au transfert des porteurs vers l'AlGaAs. En conséquence, le courant grille est négligeable et l'évolution du courant drain en fonction de la tension source-drain (Fig. 4) reste identique à celle d'un Misfet classique (Isolant/Semiconducteur).

Les figures 5 et 6 nous montrent que pour une tension de grille fortement positive $\left(V_{\mathrm{gl}}=2,0 \mathrm{~V}\right)$ et pour des tensions de drain supérieures à $0,5 \mathrm{~V}$, l'énergie moyenne des

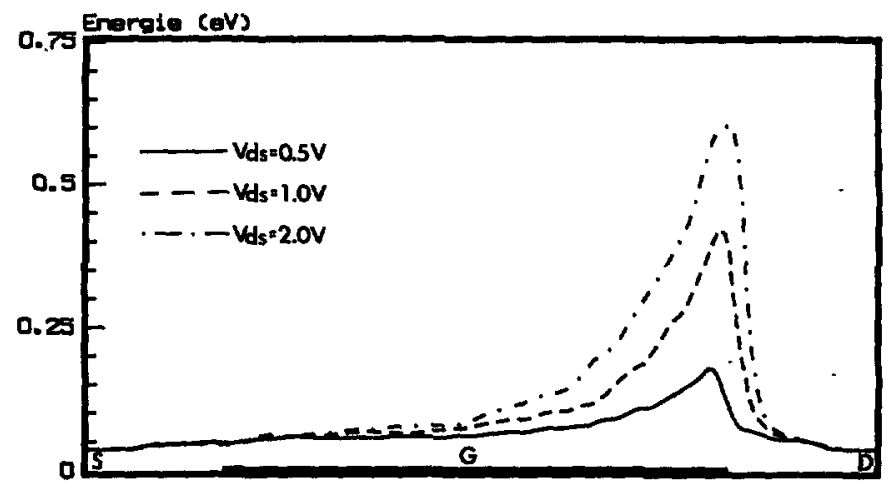

Fig. 2. - Energie moyenne des électrons à l'interface le long de l'axe source-drain à faible tension grille $\left(V_{\mathrm{g} 1}=0,4 \mathrm{~V}\right)$ pour différentes valeurs de $V_{\mathrm{ds}}$.

[Mean electron energy at the interface along source-drain axis at low gate bias $\left(V_{\mathrm{g}}=0.4 \mathrm{~V}\right)$ for several $V_{\mathrm{ds}}$.

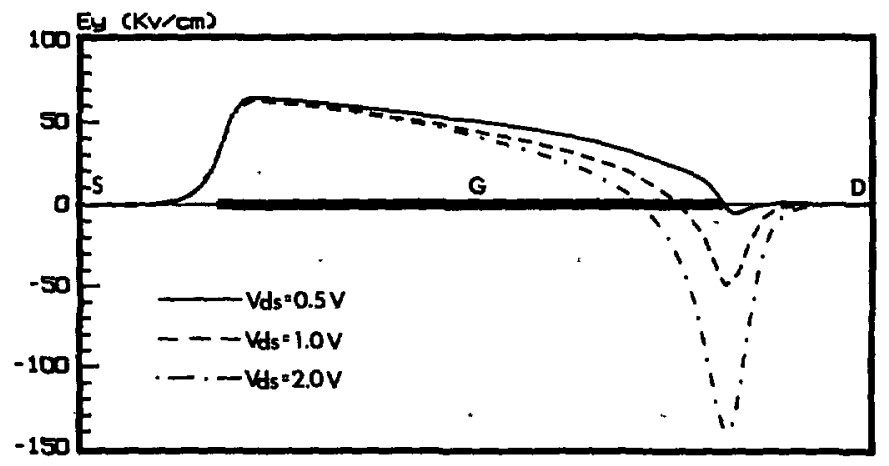

Fig. 3. - Champ électrique perpendiculaire à l'interface le long de laxe source-drain à faible tension grille $\left(V_{\mathrm{g}}=0,4 \mathrm{~V}\right)$ pour différentes valeurs de $V_{\mathrm{ds}}$.

[Transverse electric field at the interface along source-drain axis at low gate bias $\left(V_{g}=0.4 \mathrm{~V}\right)$ for several $V_{\mathrm{ds}}$.] 


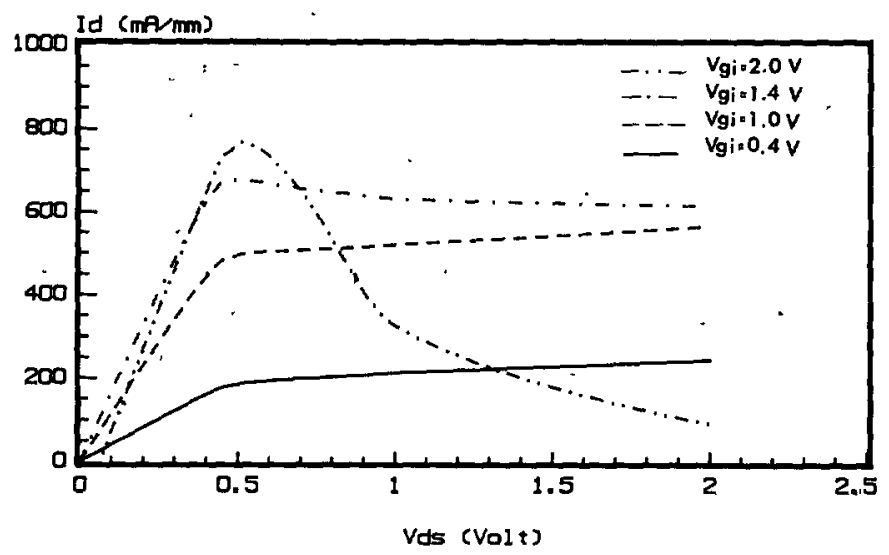

Fig. 4. - Courant de drann $I_{\mathrm{d}}$ en fonction de $V_{\mathrm{ds}}$ pour différents $V_{\mathrm{g} 1}$ de la structure MIS-Like-FET autoalignée simulée $\left(L_{\mathrm{sg}}=L_{\mathrm{gd}}=0,0 \mu \mathrm{m}\right)$.

[Drain current $I_{\mathrm{d}}$ versus $V_{\mathrm{ds}}$ for several bias $V_{\mathrm{g}}$ for simulated self-aligned MIS-Like-FET structure $\left(L_{\mathrm{sg}}=L_{\mathrm{gd}}=0.0 \mu \mathrm{m}\right)$.]

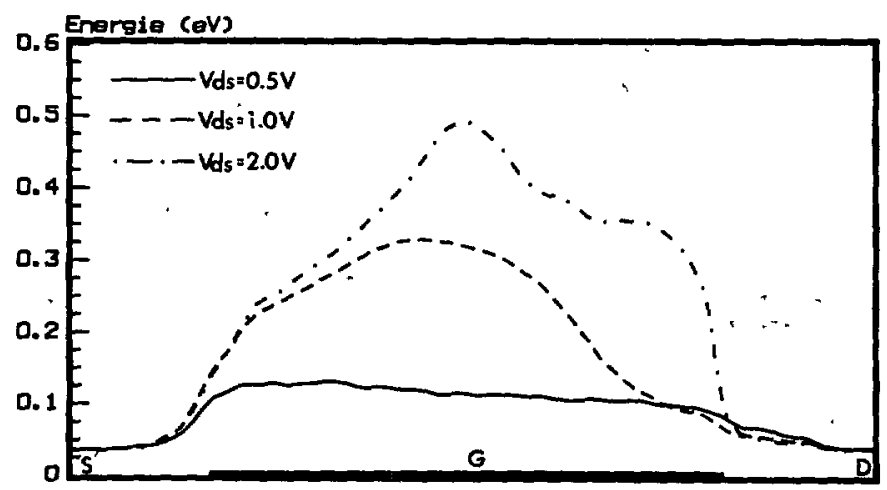

Fig. 5. - Energie moyenne des électrons à l'interface le long de l'axe source-drain à forte tension grille $\left(V_{\mathrm{g}}=2,0 \mathrm{~V}\right)$ pour différentes valeurs de $V_{\mathrm{ds}}$.

[Mean electron energy at the interface along source-drain axis at high gate bias $\left(V_{\mathrm{g}}=2.0 \mathrm{~V}\right)$ for several $\left.V_{\mathrm{ds}}.\right]$

porteurs devient supérieure à $\Delta E_{\mathrm{c}}$, avec un champ perpendiculaire à l'interface important et positif qui favorise un mouvement des porteuŕs vers la grille, ils ont donc la possibilité de franchir la barrière de potentiel et de contribuer au courant grille. Ce courant va diminuer le courant drain et nous aurons ainsi apparition d'un phénomène de résistance différentielle négative. Ce phénomène est illustré sur la figure 4 , il. s'accentue lorsque la tension grille augmente. Il est à noter que le transfert des porteurs vers la grille et surtout important en début de grille à cause du champ perpendiculaire qui y est plus important.

\section{Etude d'une structure non auto-alignée.}

Il est difficile technologiquement de fabriquer une structure parfaitement auto-alignée, nous avons donc simulé une structure non auto-alignée, avec $L_{\mathrm{sg}}=L_{\mathrm{gd}}=0,1 \mu \mathrm{m}$ et une longueur de grille de $1 \mu \mathrm{m}$. 


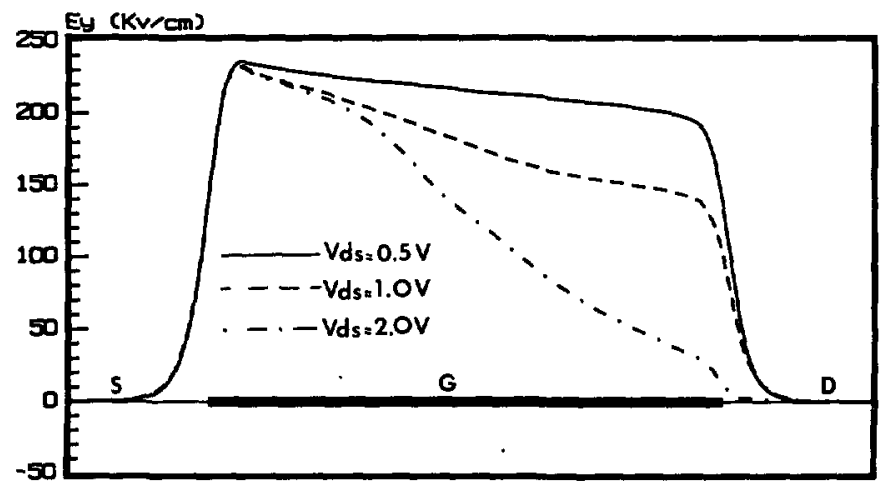

Fig. 6. - Champ électrique perpendiculaire à l'interface le long de l'axe source-drain à forte tension grille $\left(V_{\mathrm{g} 1}=2,0 \mathrm{~V}\right)$ pour différentes valeurs de $V_{\mathrm{ds}}$.

[Transverse electric field at the interface along source-drain axis at high gate bias $\left(V_{\mathrm{g} 1}=2.0 \mathrm{~V}\right)$ for several $\left.V_{\mathrm{ds}}\right]$

Nous remarquons sur la figure 7 que pour une tension grille de $0,4 \mathrm{~V}$, le courant drain est plus faible que pour une structure auto-alignée. Ceci s'explique par l'action de la grille, plus éloignée des contacts ("réservoir » d'électrons) qui entraîne une diminution de la charge $N_{s}$ accumulée à l'interface.

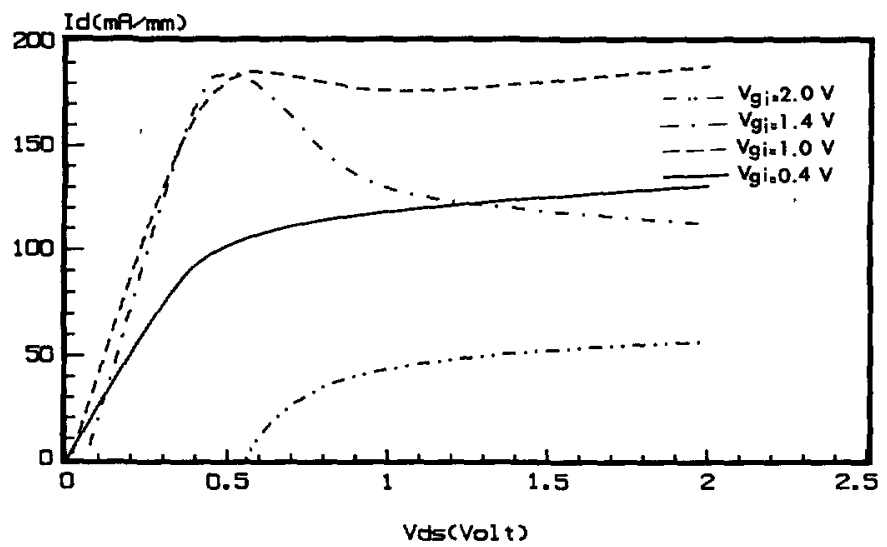

Fig. 7. - Courant drain $I_{\mathrm{d}}$ en fonction de $V_{\mathrm{ds}}$ pour différents $V_{\mathrm{g}}$ de la structure MIS-Like-FET simulée avec $L_{\mathrm{sg}}=L_{\mathrm{gd}}=0,1 \mu \mathrm{m}$.

[Drain current $I_{\mathrm{d}}$ versus $V_{\mathrm{ds}}$ for several bias $V_{\mathrm{gg}}$ for MIS-Like-FET structure with $L_{\mathrm{sg}}=L_{\mathrm{gd}}=0.1 \mu \mathrm{m}$.]

Lorsque $V_{\mathrm{gi}}$ est égale à $1,4 \mathrm{~V}$, le courant grille augmente de façon plus importante dans le cas présent, dès les tensions $V_{d s}$ faibles (Fig. 8). En effet l'espace source-grille fait apparaître, comme nous le constatons sur la figure 9 , une composante de champ parallèle à l'interface, plus élevée en début de grille, qui échauffe les porteurs et leur permet de transférer en plus grand nombre vers l'AlGaAs. Le courant grille prend des valeurs plus importantes aux faibles tensions $V_{\mathrm{ds}}$ (Fig. 8) et son augmentation avec $V_{\mathrm{ds}}$ est moindre, ce qui se traduit par une 


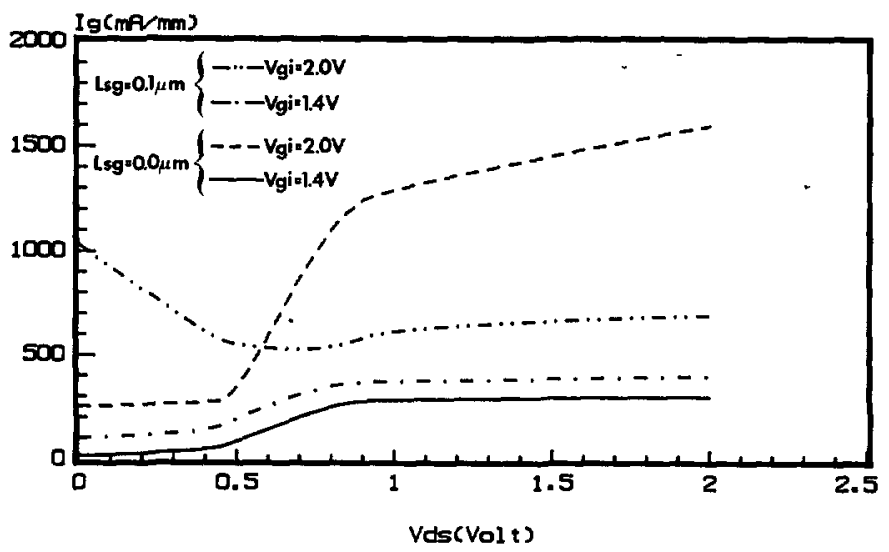

Fig. 8. - Courant grille $I_{\mathrm{g}}$ en fonction de $V_{\mathrm{ds}}$ à fortes tensions de grille $\left(V_{\mathrm{g}}=1,4-2,0 \mathrm{~V}\right)$ pour $L_{\mathrm{sg}}=L_{\mathrm{gd}}=0,0 \mu \mathrm{m}$ et $L_{\mathrm{sg}}=L_{\mathrm{gd}}=0,1 \mu \mathrm{m}$.

[Gate current $I_{\mathrm{g}}$ versus $V_{\mathrm{ds}}$ for high gate bias $\left(V_{\mathrm{gl}}=1.4-2.0 \mathrm{~V}\right)$ for $L_{\mathrm{sg}}=L_{\mathrm{gd}}=0.0 \mu \mathrm{m}$ and $\left.L_{\mathrm{sg}}=L_{\mathrm{gd}}=0.1 \mu \mathrm{m}.\right]$

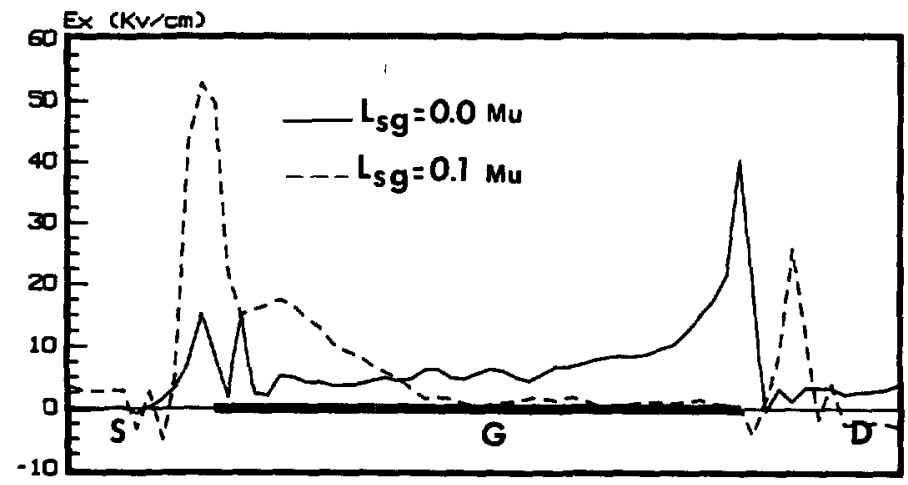

Fig. 9. - Champ électrique longitudinal à l'interface le long de l'axe source-drain à $V_{\mathrm{g}}=1,4 \mathrm{~V}$, $V_{\mathrm{ds}}=0,5 \mathrm{~V}$ pour $L_{\mathrm{sg}}=L_{\mathrm{gd}}=0,1 \mu \mathrm{m}$ et $L_{\mathrm{sg}}=L_{\mathrm{gd}}=0,0 \mu \mathrm{m}$.

[Longitudinal electric field at the interface along source-drain axis at $V_{\mathrm{gt}}=1.4 \mathrm{~V}, V_{\mathrm{ds}}=0.5 \mathrm{~V}$ for $L_{\mathrm{sg}}=L_{\mathrm{gd}}=0.0 \mu \mathrm{m}$ and $L_{\mathrm{sg}}=L_{\mathrm{gs}}=0.1 \mu \mathrm{m}$.]

conductance différentielle moins élevée. Pour les fortes tensions grille $\left(V_{\mathrm{gi}}=2,0 \mathrm{~V}\right)$ le courant grille est très important dès les faibles tensions $V_{\mathrm{ds}}\left(V_{\mathrm{ds}}<0,5 \mathrm{~V}\right)$, et l'on constate que le courant drain est négatif. Ceci s'explique par un transfert important vers la grille des électrons provenant de la source et du drain comme l'illustre la figure 10.

\section{Influence de la résistance de grille $\boldsymbol{R}_{\mathrm{g}}$.}

La résistance du contact de grille est un paramètre qui influe de façon non négligeable sur l'amplitude de la résistance différentielle négative. Pour étudier son effet, nous avons tenu compte dans notre modèle de la résistance de grille $R_{\mathrm{g}}$ en appliquant la relation suivante :

$$
V_{\mathrm{gs}}=V_{\mathrm{gi}}+\Phi_{\mathrm{b}}+R_{\mathrm{g}} \cdot I_{\mathrm{g}}
$$




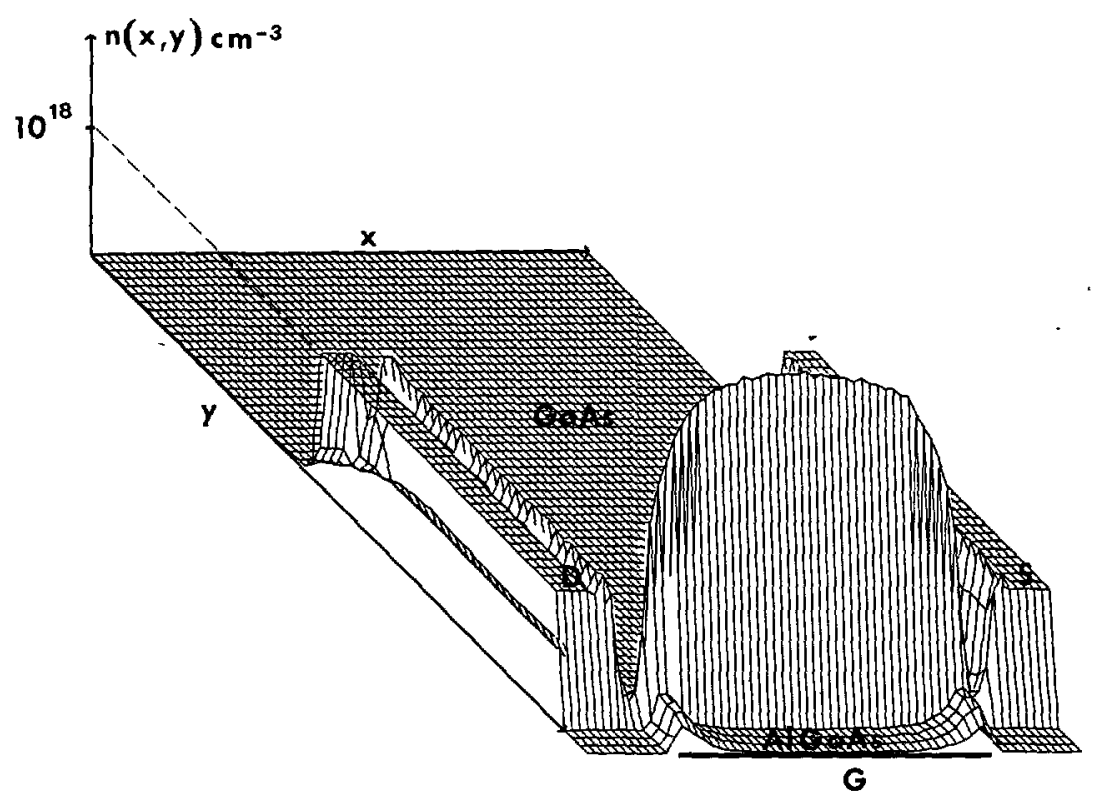

Fig. 10. - Représentation tridimensionnelle de la densité électronique ̀̀ $V_{\mathrm{g}}=2,0 \mathrm{~V}, \quad V_{\mathrm{ds}}=0,0 \mathrm{~V}$, $L_{\mathrm{sg}}=L_{\mathrm{gd}}=0,1 \mu \mathrm{m}$. On voit bien que le transfert des porteurs de la zone d'accumulation vers la grille se fait surtout en début et fin de grille.

[3D plot of electron density showing real space transfer of electron at source and drain side of the gate $\left.\left(V_{\mathrm{gl}}=2.0 \mathrm{~V}, V_{\mathrm{ds}}=0.0 \mathrm{~V}, L_{\mathrm{sg}}=L_{\mathrm{gd}}=0.1 \mu \mathrm{m}.\right)\right]$

où $V_{g l}$ est la tension grille sur le semiconducteur, $V_{g s}$ est la tension appliquée sur la grille, $I_{\mathrm{g}}$ est le courant grille calculé. Nous avons choisi pour une $R_{\mathrm{g}}$ une valeur «typique » de $1 \Omega$ par $\mathrm{mm}$ de grille. Nous voyons figure 11 que la conductance différentielle négative a une

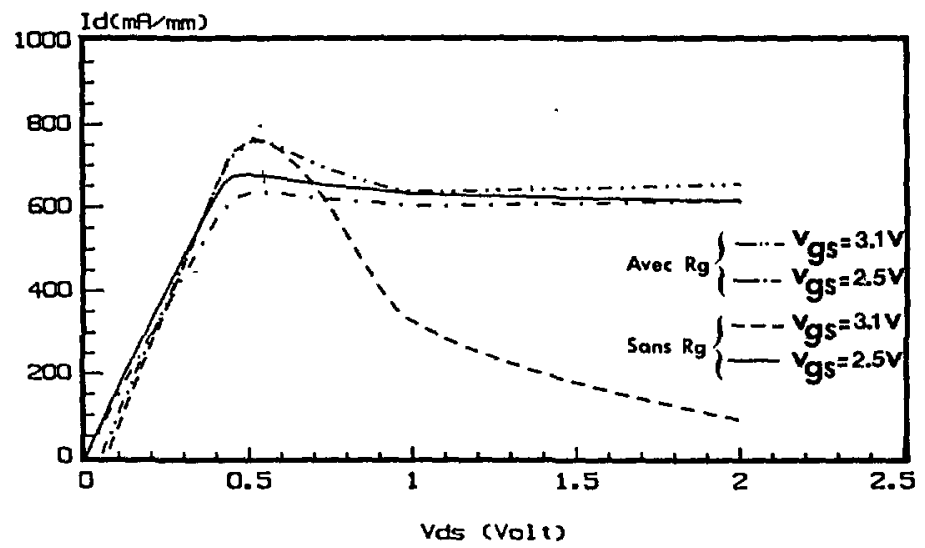

Fig. 11. - Courant drain en fonction de $V_{\mathrm{ds}}$ à fortes tensions de grilles $\left(V_{\mathrm{gs}}=2,5-3,1 \mathrm{~V}\right)$ pour $R_{\mathrm{g}}=0 \Omega / \mathrm{mm}$ et $R_{\mathrm{g}}=1 \Omega / \mathrm{mm}$ avec $L_{\mathrm{sg}}=L_{\mathrm{gd}}=0.0 \mu \mathrm{m} \quad$ (N.B. $V_{\mathrm{gs}}=V_{\mathrm{g}}+\Phi_{\mathrm{b}}+R_{\mathrm{g}} \cdot I_{\mathrm{g}}$ avec $\Phi_{\mathrm{b}}=1,1 \mathrm{eV}$ ).

[Drain current versus $V_{\mathrm{ds}}$ for high gate bias $\left(V_{\mathrm{gs}}=2.5-3.1 \mathrm{~V}\right.$ ) for $R_{\mathrm{g}}=0 \Omega / \mathrm{mm}$ and $R_{\mathrm{g}}=1 \Omega / \mathrm{mm}$, $L_{\mathrm{sg}}=L_{\mathrm{gd}}=0.0 \mu \mathrm{m}$ (N.B. $V_{\mathrm{gs}}=V_{\mathrm{g}}+\Phi_{\mathrm{b}}+R_{\mathrm{g}} \cdot I_{\mathrm{g}}$ with $\Phi_{\mathrm{b}}=1.1 \mathrm{eV}$ ).] 
amplitude beaucoup plus faible par rapport au cas où $R_{\mathrm{g}}$ est nulle. La résistance de grille intervient quand le courant grille est important car elle crée une chute de potentiel $R_{\mathrm{g}} . I_{\mathrm{g}}$ qui va réduire le courant grille. Ainsi le courant drain ne sera pas trop diminué.

\section{Influence du dopage du canal.}

Une des principales limitations du MIS-Like-FET est la faible valeur du courant drain qui « interdit » les applications en puissance. Une solution pour pallier à cet inconvénient est de doper la zone active du GaAs, ce qui conduit à considérer un nouveau composant dénommé Doped-Channel MIS-Like-FET (DMT) [9]. Ce composant peut fonctionner en déplétion, comme un Mesfet $\left(V_{\mathrm{g}}<0\right)$ ou en enrichissement comme le MIS-Like-FET. Pour illustrer l'intérêt potentiel de ce composant, nous avons utilisé notre modèle pour calculer les caractéristiques $I_{\mathrm{d}}\left(V_{\mathrm{ds}}, V_{\mathrm{gs}}\right)$ d'un DMT comportant $200 \AA$ de GaAs dopé à $3 \times 10^{18} \mathrm{~cm}^{-3}$,

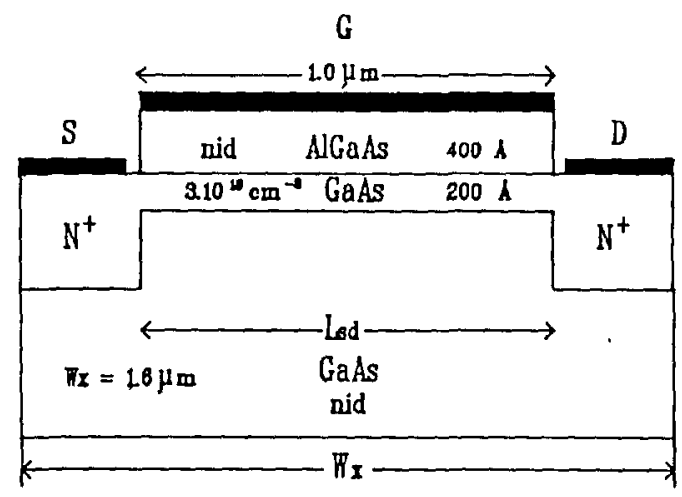

Fig. 12. - Structure DMT simulée.

[Simulated structure of DMT.]

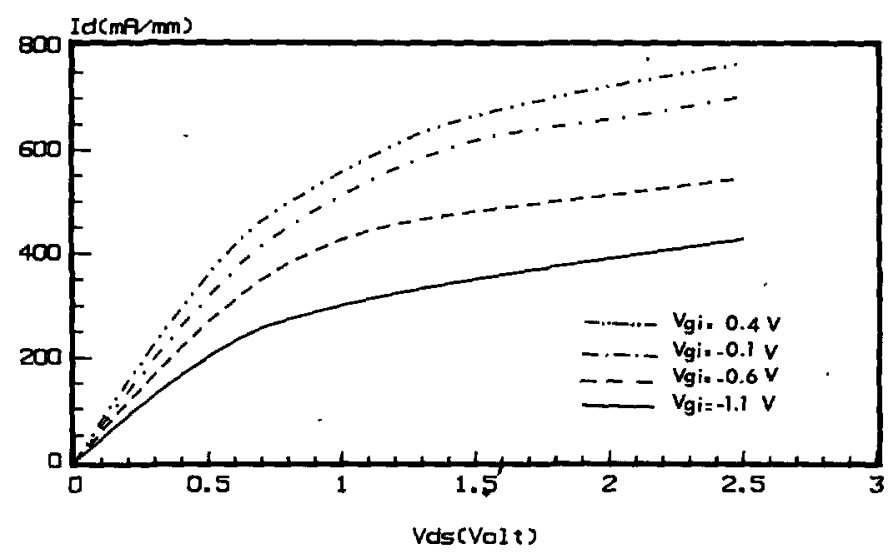

Fig. 13. - Courant drain en fonction de $V_{\mathrm{ds}}$ pour différentes valeurs de tension grille pour la structure DMT.

[Drain current versus $V_{\mathrm{ds}}$ for several bias $V_{\mathrm{gt}}$ for DMT structure.] 
les autres paramètres sont décrits figure 12. Nous constatons d'après la figure 13 que pour une tension de grille de $0,4 \mathrm{~V}$ le courant drain en saturation est considérablement plus important pour le DMT $(750 \mathrm{~mA} / \mathrm{mm})$ que pour le MIS-Like-FET auto-aligné $(200 \mathrm{~mA} / \mathrm{mm})$. Ceci confirme l'intérêt de cette structure pour les applications nécessitant de fortes valeurs de courant commandé.

\section{Conclusion.}

Cette étude a permis de mettre en évidence les phénomènes de courant grille et de résistance différentielle négative et de décrire les mécanismes physiques qui en sont à l'origine. Nous avons également étudié l'influence de divers paramètres sur le comportement du composant. Nous avons montré que l'amplitude de la conductance différentielle négative est très sensible à certains paramètres technologiques tels que la distance source-grille et la résistance de la métallisation de grille, ces deux quantités devant être réduites le plus possible. Si l'on s'intéresse au fonctionnement "classique " (bloqué-saturé), le critère d'optimisation sera d'obtenir un courant de drain suffisant en évitant l'apparition du courant grille. Nous avons montré aussi que le dopage du canal permettait d'augmenter ce courant drain et de parvenir à ce but. Une autre solution envisageable serait l'utilisation d'un couple de matériaux tel que AlInAs/GaInAs, pour lequel la valeur élevée de la hauteur de barrière devrait limiter le courant grille.

\section{Bibliographie}

[1] KASZYNSKY A., Thèse de Docteur Ingénieur (Lille, 1979).

[2] HOCKNEY R. W. et al., Electron. Lett. 10 (1974) 484.

[3] Brooks H., Advan. Electron. Phys. 7 (1955) 85.

[4] Adachi S., J. Appl. Phys. 58 (1985) R1-R29.

[5] Drummond T. J. et al., Appl. Phys. Lett. 41 (1982) 277.

[6] Drummond T. J. et al., I.E.E.E. Electron. Dev. Lett. EDL-3 (1983) 207.

[7] DePreeuw D., Thèse de docteur de l'Université (Lille, 1988).

[8] BeST J. S., Appl. Phys. Lett. 34 (1979) 522.

[9] HidA H. et al., I.E.E.E. Trans. Electron. Dev. ED-34 (1987) 1448. 